\title{
ANÁLISIS DE LA RESPUESTA ESTRUCTURAL DE PAVIMENTOS MEDIANTE DEFLECTOMETRÍA DE IMPACTO (FWD) EN DIFERENTES ÉPOCAS DEL AÑO EN COSTA RICA
}

\author{
Gustavo A. Badilla Vargas
} Denia Sibaja Obando

\begin{abstract}
Resumen
El Laboratorio Nacional de Materiales y Modelos Estructurales (LanammeUCR) de la Universidad de Costa Rica, realiza cada dos años una evaluación de la red vial nacional pavimentada (ERVN), la cual sirve como instrumento para la gestión de dicha infraestructura.

Tomando en cuenta que los datos de deflectometría de impacto (FWD) podría tomarse en diferentes épocas del año, se plantea la necesidad de cuantificar la variación de los resultados producto de los cambios de humedad de las capas granulares y suelos, relacionados con la estación climática.

Para verificar la existencia de variaciones en la respuesta estructural, reflejada en la deflexión en el primer sensor del FWD, asociado con la estación climática (lluviosa y seca), se realizó un análisis estadístico a la base de datos de resultados de FWD, recopilados en treinta y dos puntos diversos en campañas de medición realizadas en diferentes épocas entre el 2003 y el 2006 en seis regiones geográficas del país.

Los resultados muestran que las estructuras sometidas a cambios marcados del contenido de humedad presentan variaciones significativas en la respuesta. Por el contrario, las zonas en las cuales no existen cambios importantes en el contenido de humedad (más de $180 \mathrm{~d}$ de lluvia en el año) no presentan diferencias significativas entre los resultados.
\end{abstract}

Palabras claves: deflectometría de impacto, FWD, zonificación climática, respuesta estructural, pavimentos.

\begin{abstract}
Every two years, the Laboratorio Nacional de Materiales y Modelos Estructurales (LanammeUCR) of the Universidad de Costa Rica carries out an Evaluation of the Paved National Road Network which is a tool to the Asset Management.

The Falling Weight Deflectometer (FWD) can be used on different times of the year. Consequently, it is necessary to quantify the variation of results due to changes in humidity on granular layers or soils associated with the seasonal variation.

In order to test the existence of structural response variations reflected in the value of the first sensor's FWD associated with the climatic season (dry and rainy), a statistical analysis of the FDW results database was made in thirty two different points located in six geographic zones around the country. For each point measurements were made in different times between 2003 and 2006.

The result shows that pavements with significant changes of content of humidity have significant variations of structural response. On the other hand, the zones with insignificant changes of content of humidity (more than 180 rainy days on the year) don't show significant changes in results.
\end{abstract}

Keywords: falling weight deflectometer, FWD, climatic zoning, structural response, pavements.

Recibido: 23 de noviembre de 2009 •Aprobado: 08 de junio de 2010

\section{ALCANCE}

Para la realización de este estudio se cuenta con las mediciones del D1 (deflexión medida bajo el centro del plato de carga del deflectómetro de impacto) de treinta y dos diferentes puntos localizados en seis regiones geográficas y en once zonas climáticas del país, para los cuales se conoce la estructura del pavimento (espesores) y materiales que los conforman.

El estudio comprende la comparación de la deflexión D1 obtenida mediante el deflectómetro de impacto, como medida indirecta del efecto 
de reducción, producto de la humedad en la que se encuentran las capas granulares y suelos. El análisis de la variación estacional fue verificada para aquellos puntos en los que se realizaron mediciones en las distintas estaciones climáticas en el año, ya que de lo contrario, no sería posible evaluar la influencia de la variación de la humedad en la respuesta estructural del pavimento.

Se reconoce que la estructura del pavimento influye directamente en su respuesta estructural; estructuras robustas (de mayores espesores y módulos resilientes altos) presentarán deflexiones menores.

Se considerará en este estudio el espesor de la carpeta asfáltica, que se toma en cuenta para realizar los cálculos necesarios para corregir los datos de deflectometría por temperatura. El método utilizado para realizar la corrección por temperatura es el propuesto por el Asphalt Institute y la guía de diseño (AASHTO, 1993) el cual toma en cuenta el espesor de la carpeta, la temperatura de la superficie y la del aire.
Para definir si existe variación en los valores de las deflexiones medidas en diferentes estaciones climáticas, se emplearán herramientas estadísticas para comparar los promedios de las deflexiones medidas cada $10 \mathrm{~m}$ con el sensor D1, en un tramo de $220 \mathrm{~m}$ para cada punto de análisis, distribuidos en las distintas regiones del país y ubicados cerca de estaciones meteorológicas.

\section{METODOLOGÍA}

Para realizar esta investigación se procedió a seguir la secuencia de actividades mostrada en la Figura 1.

Para ubicar la ubicación de los puntos evaluados con el deflectómetro de impacto, el LanammeUCR cuenta con una base de datos de resultados del deflectómetro de impacto (FWD) recopilados en treinta y dos puntos localizados en seis regiones geográficas del país, en los cuales

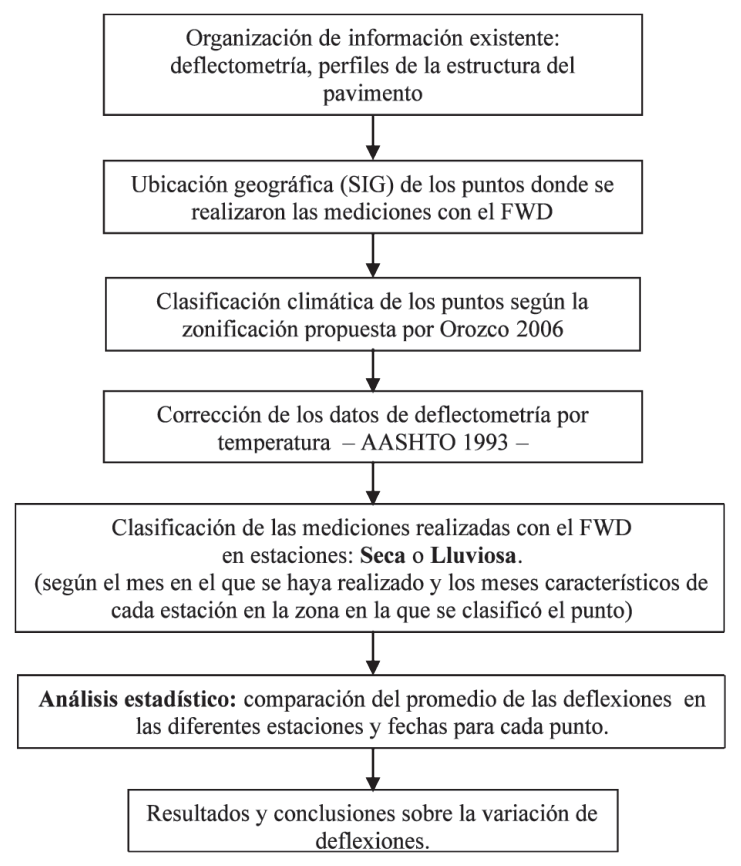

Figura 1. Metodología utilizada.

Fuente: (El autor y la autora). 
se realizaron diferentes campañas de medición, en diferentes épocas, entre el 2003 y el 2006.

Para cada uno de los puntos seleccionados, se realizó una excavación a cielo abierto para determinar los espesores de cada una de las capas, además, se realizaron ensayos de laboratorio a los materiales constituyentes. La metodología de medición consistió en hacer ensayos con el deflectómetro de impacto (FWD) cada $10 \mathrm{~m}$. Estos ensayos se hicieron 120 $\mathrm{m}$ antes y $120 \mathrm{~m}$ después del punto donde se realizó la excavación. Para los materiales de la base, subbase y subrasante se hicieron ensayos de: Próctor (estándar y modificado), límites de Atterberg, granulometrías, CBR.

Como primer paso se procedió a realizar la ubicación geográfica de los treinta y dos puntos y posteriormente asignarle las características climáticas correspondientes a la zonificación climática propuesta por (Orozco, 2007); en el Cuadro 1 se presenta un resumen de la zonificación climática propuesta para Costa Rica, así como los meses característicos para cada estación por zona climática.

Para realizar el análisis de los datos se consideró que la condición predominante para los meses de transición era la correspondiente a la estación inmediatamente anterior. Por ejemplo, en el caso de la Zona Climática CGT, se tiene que abril es el mes de transición de la estación seca a la lluviosa, por lo tanto se considera para el análisis, que abril conserva las características de la estación seca. Por otro lado, diciembre es el mes de transición de la estación lluviosa a la seca, por lo tanto se considera que diciembre conserva las características de la estación lluviosa.

En la Figura 2 se muestra la ubicación de los puntos en las distintas zonas climáticas. Con base en la Figura 2 y el Cuadro 1 es posible definir la estación seca y la estación lluviosa en la que fueron tomados los datos de deflectometría para

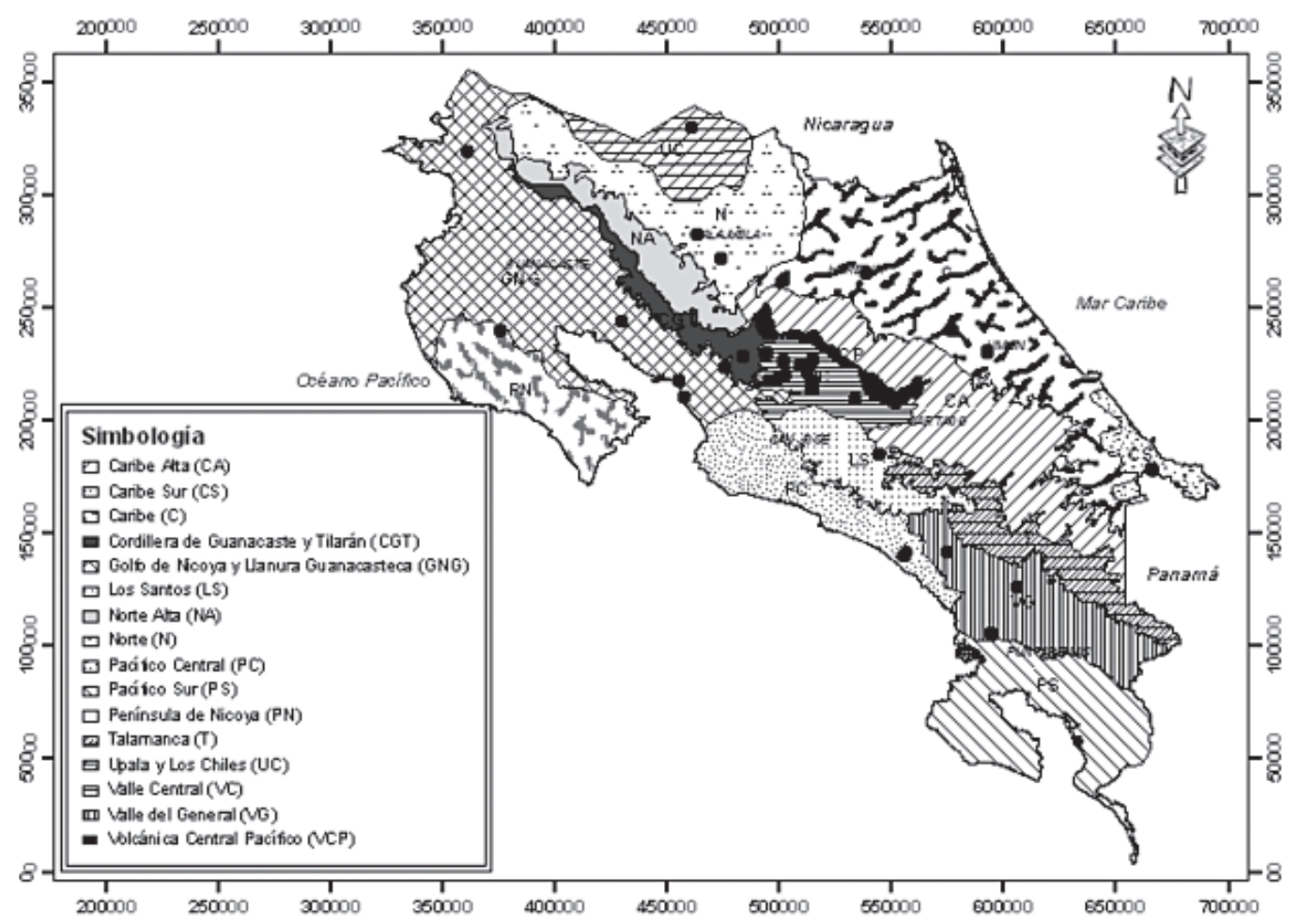

Figura 2. Ubicación de puntos para las zonas climáticas definidas por (Orozco, 2007). 
Cuadro 1. Zonas climáticas de Costa Rica y meses característicos.

Zona

Caribe

Caribe Alta

Cordillera Guanacaste -

Tilarán

Caribe Sur

Golfo Nicoya - Llanura

Guanacaste

Los Santos - Caribe Alta

Norte

Norte Alta

Pacífico Central

Península de Nicoya

Pacífico Sur

Talamanca

Upala y Los Chiles

Valle Central

Volcánica Central Pacífico

Valle del General

\section{Símbolo}

C

CA

CGT

CS

GNG

LS

$\mathrm{N}$

NA

PC

PN

PS

$\mathrm{T}$

UC

enero, febrero,

VC

VCP

VG marzo

enero, febrero, marzo

diciembre, enero, febrero, marzo

diciembre, enero, febrero, marzo

diciembre, enero, febrero, marzo

enero, febrero,

marzo

abril

enero, febrero,

marzo

diciembre marzo, abril

diciembre

diciembre, enero,

febrero, marzo,

abril

setiembre

setiembre

abril, diciembre

marzo, setiembre

abril, noviembre

abril, noviembre

marzo

febrero, abril

abril, diciembre

abril, noviembre

enero, febrero, marzo

enero, febrero, marzo

\section{Época lluviosa}

marzo, abril, mayo, junio, julio, agosto, octubre, noviembre, diciembre, enero, febrero

marzo, abril, mayo, junio, julio, agosto, octubre, noviembre, diciembre, enero, febrero

mayo, junio, julio, agosto, setiembre, octubre, noviembre

abril, mayo, junio, julio, agosto, octubre, noviembre, diciembre, enero, febrero

mayo, junio, julio, agosto, setiembre, octubre

mayo, junio, julio, agosto, setiembre, octubre

abril, mayo, junio, julio, agosto, setiembre, octubre, noviembre, diciembre, enero, febrero

mayo, junio, julio, agosto, setiembre, octubre, noviembre, diciembre, enero

mayo, junio, julio, agosto, setiembre, octubre, noviembre

mayo, junio, julio, agosto, setiembre, octubre

mayo, junio, julio, agosto, setiembre, octubre, noviembre, diciembre

abril, mayo, junio, julio, agosto, setiembre, octubre, noviembre

mayo, junio, julio, agosto, setiembre, octubre, noviembre

mayo, junio, julio, agosto, setiembre, octubre

mayo, junio, julio, agosto, setiembre, octubre, noviembre

mayo, junio, julio, agosto, setiembre, octubre, noviembre

Fuente: (El autor y la autora). 
cada uno de los puntos, el resumen se presenta en el Cuadro 2.

Como se puede observar en el Cuadro 1, es de esperar que para algunas zonas, a pesar de que se realicen mediciones en distintos meses del año, como se observa en el Cuadro 2, todas las mediciones correspondan a meses lluviosos. Esto se debe a que en algunas de estas regiones la época lluviosa comprende la totalidad del año o un gran porcentaje de este.

\subsection{Corrección de deflectometría por temperatura}

El Instituto de Asfalto estableció un método para la corrección de las deflexiones en función de la temperatura de la capa asfáltica. El procedimiento consiste en:

1. Determinar la temperatura promedio de la capa asfáltica: Para ello se supone un gradiente lineal de temperatura; por la tanto, la temperatura media de la capa asfáltica será el promedio entre la temperatura superficial y la de la fibra inferior. La temperatura del pavimento viene dada por la ecuación (1).

$$
\mathrm{t}_{\text {pavimento }}=\frac{1}{2}\left(\mathrm{t}_{\text {sup.pav }}+\mathrm{t}_{\text {inf.pav }}\right)
$$

Donde:

$\mathrm{t}_{\text {pavimento }}$ temperatura promedio del pavimento $\left({ }^{\circ} \mathrm{C}\right)$

$t_{\text {sup. pav temperatura de la superficie del }}$ pavimento en el ensayo $\left({ }^{\circ} \mathrm{C}\right)$

$t_{\text {inf. pav temperatura en la fibra inferior }}$ del pavimento $\left({ }^{\circ} \mathrm{C}\right)$, estimada de la Figura 3.

Nótese que es necesario conocer la temperatura superficial del pavimento en el ensayo y la temperatura media del aire de los $5 \mathrm{~d}$ previos al ensayo, así como el espesor de la capa asfáltica.

2. Determinar el factor de corrección por temperatura: Utilizando el valor de la temperatura promedio de la capa calculado anteriormente, se determina el factor de corrección por temperatura, para ello se

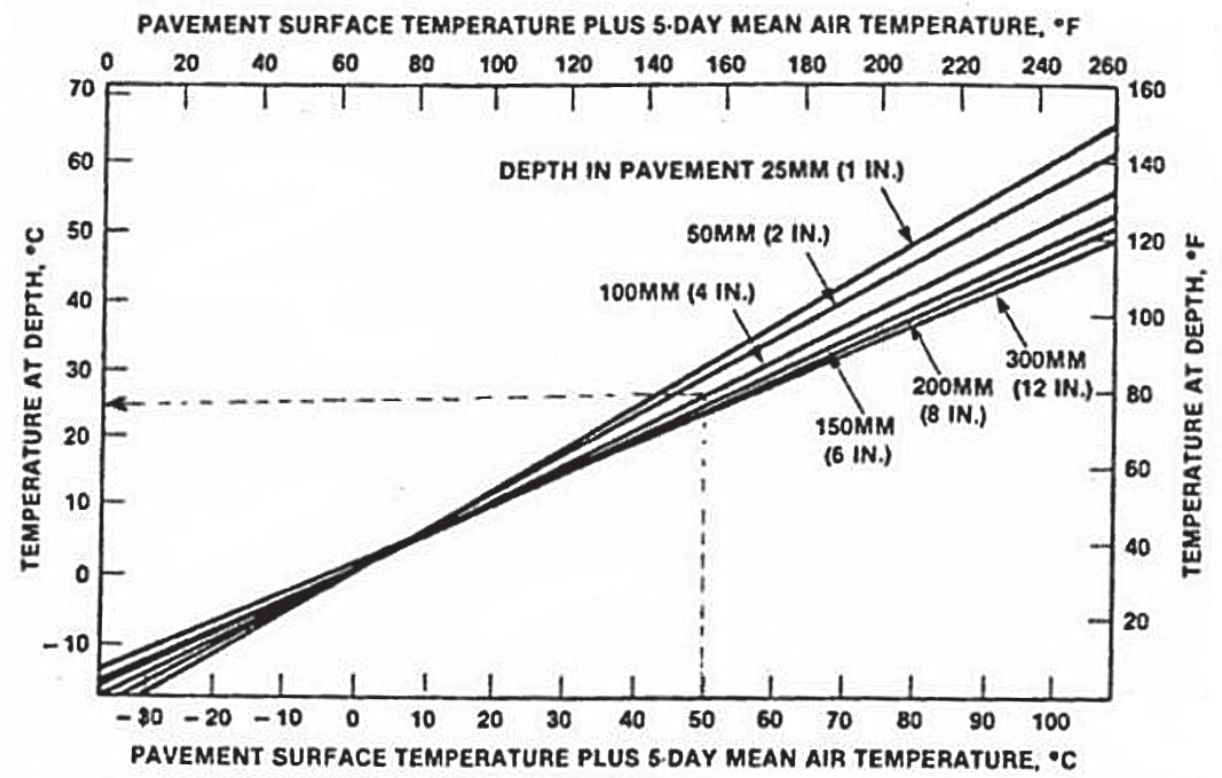

Figura 3. Temperatura en función del espesor de la capa asfáltica Fuente: (Asphalt Institute, s.f.). 
Cuadro 2. Resumen de mediciones realizadas con el FWD.

\begin{tabular}{|c|c|c|c|}
\hline Punto & Fechas FWD & Estación & Región climática \\
\hline \multirow{6}{*}{$\begin{array}{c}\text { Punto 1. } \\
\text { Monterrey - Jicarito }\end{array}$} & $21-10-03$ & Lluviosa & \multirow{5}{*}{ Norte } \\
\hline & 20-01-04 & Lluviosa & \\
\hline & $20-10-04$ & Lluviosa & \\
\hline & $25-01-05$ & Lluviosa & \\
\hline & $25-04-06 *$ & Lluviosa & \\
\hline & $22-10-03$ & Lluviosa & \multirow{6}{*}{ Caribe } \\
\hline \multirow{4}{*}{$\begin{array}{c}\text { Punto 2. } \\
\text { Aguas Zarcas - Venecia }\end{array}$} & 21-01-04 & Lluviosa & \\
\hline & $19-10-04$ & Lluviosa & \\
\hline & $24-01-05$ & Lluviosa & \\
\hline & $24-07-06 *$ & Lluviosa & \\
\hline \multirow{6}{*}{$\begin{array}{c}\text { Punto } 3 . \\
\text { Tanque - Florencia }\end{array}$} & $22-10-03$ & Lluviosa & \\
\hline & $20-01-04$ & Lluviosa & \multirow{6}{*}{ Norte } \\
\hline & $20-10-04$ & Lluviosa & \\
\hline & $25-01-05$ & Lluviosa & \\
\hline & $25-07-06^{*}$ & Lluviosa & \\
\hline & $22-10-03$ & Lluviosa & \\
\hline \multirow{4}{*}{$\begin{array}{c}\text { Punto } 4 . \\
\text { Ciudad Quesada - Los Chiles }\end{array}$} & 21-01-04 & Seca & \\
\hline & $19-10-04$ & Lluviosa & \multirow[t]{3}{*}{ Upala - Los Chiles } \\
\hline & $24-01-05$ & Seca & \\
\hline & $24-07-06^{*}$ & Lluviosa & \\
\hline \multirow{4}{*}{$\begin{array}{c}\text { Punto } 5 . \\
\text { Cerro de la Muerte, km } 56\end{array}$} & 03-11-04 & Lluviosa & \multirow{4}{*}{ Los Santos } \\
\hline & $22-04-05$ & Seca & \\
\hline & $07-08-06^{*}$ & Lluviosa & \\
\hline & $10-10-03$ & Lluviosa & \\
\hline \multirow{3}{*}{$\begin{array}{c}\text { Punto } 6 . \\
\text { Barú - Dominical }\end{array}$} & $02-11-04$ & Lluviosa & \multirow{3}{*}{ Pacífico Central } \\
\hline & 07-04-05 & Seca & \\
\hline & $07-08-06 *$ & Lluviosa & \\
\hline \multirow{5}{*}{$\begin{array}{c}\text { Punto } 7 . \\
\text { Juntas Pacuar - San Isidro }\end{array}$} & $10-10-03$ & Lluviosa & \multirow{5}{*}{ Valle del General } \\
\hline & 03-11-04 & Lluviosa & \\
\hline & 08-04-05 & Seca & \\
\hline & $08-08-06^{*}$ & Lluviosa & \\
\hline & $10-10-03$ & Lluviosa & \\
\hline \multirow{3}{*}{$\begin{array}{c}\text { Punto 8. } \\
\text { Buenos Aires - Juntas del Pacuar }\end{array}$} & $02-11-04$ & Lluviosa & \multirow{3}{*}{ Valle del General } \\
\hline & 07-04-05 & Seca & \\
\hline & 08-08-06* & Lluviosa & \\
\hline
\end{tabular}

* Nota: Las fechas marcadas con asteriscos no poseen información de la temperatura del pavimento en el momento de la realización de las mediciones, por lo cual fueron descartadas para el análisis.

Fuente: (El autor y la autora, 2009). 
Cuadro 2. Resumen de mediciones realizadas con el FWD (continuación).

Punto

Río Claro - Palmar Norte

Punto 11.

Siquirres - Guácimo

Punto 12.

Siquirres - Limón

Punto 13.

Bribrí - Sixaola

Punto 14.

Manolos - La Garita
Fechas FWD

$10-10-03$

02-11-04

07-04-05

07-08-06*

27-01-04

22-10-04

02-02-05

12-07-06*

28-01-04

22-10-04

02-02-05

12-07-06*

27-01-04

21-10-04

26-01-05

12-07-06*

28-01-04

21-10-04

26-01-05

13-07-06*

13-03-02

03-11-03

13-10-04

20-04-05

17-07-06*
Estación

Lluviosa

Lluviosa

Seca

Lluviosa

Lluviosa

Lluviosa

Lluviosa

Lluviosa

Lluviosa

Lluviosa

Lluviosa

Lluviosa

Lluviosa

Lluviosa

Lluviosa

Lluviosa

Lluviosa

Lluviosa

Lluviosa

Lluviosa

Seca

Lluviosa

Lluviosa

Seca

Lluviosa
Región climática

Valle del General

Caribe

Caribe

Caribe

Caribe Sur

Valle Central

* Nota: Las fechas marcadas con asteriscos no poseen información de la temperatura del pavimento en el momento de la realización de las mediciones, por lo cual fueron descartadas para el análisis. Fuente: (El autor y la autora, 2009). 
Cuadro 2. Resumen de mediciones realizadas con el FWD (continuación).

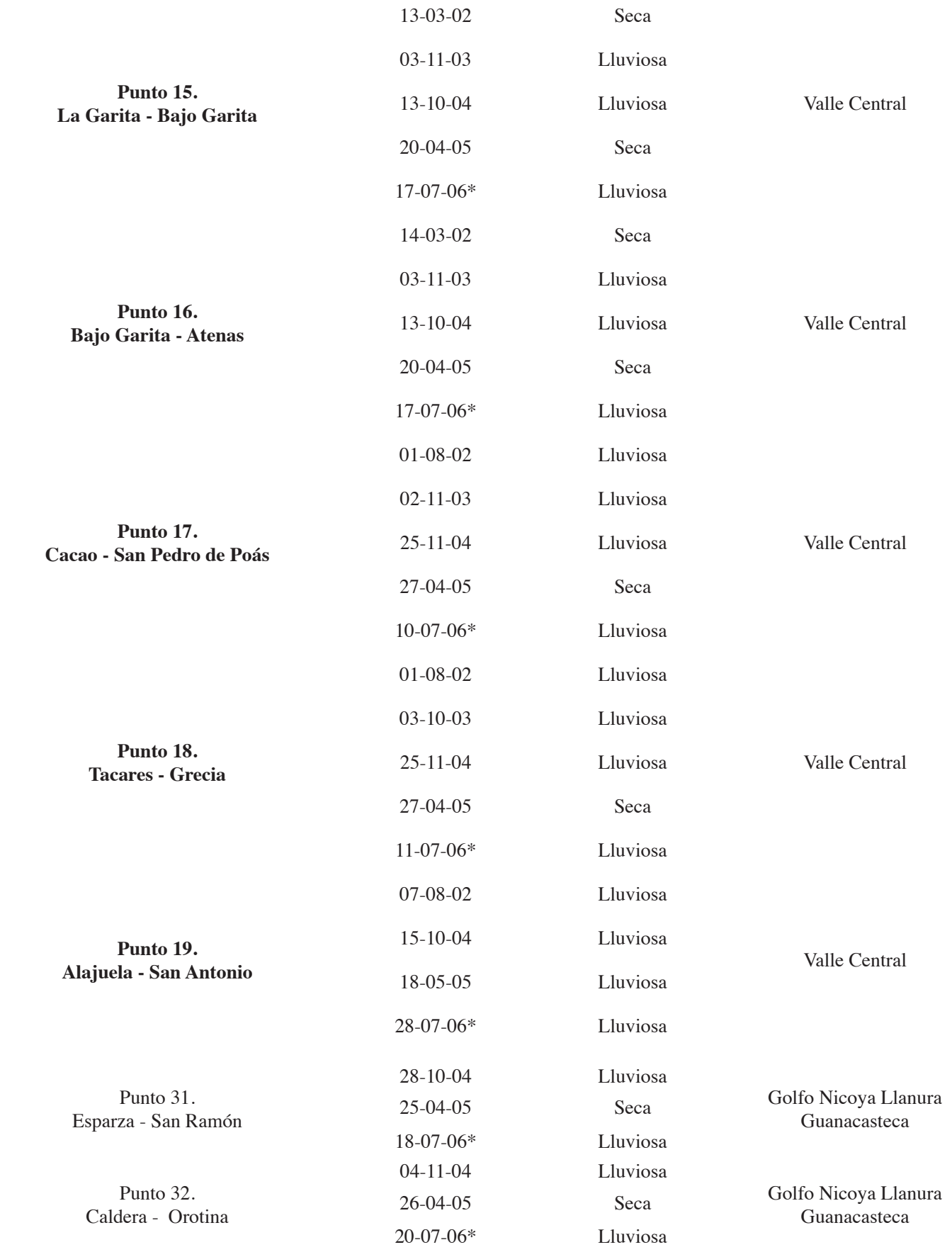

* Nota: Las fechas marcadas con asteriscos no poseen información de la temperatura del pavimento en el momento de la realización de las mediciones, por lo cual fueron descartadas para el análisis. 
Cuadro 2. Resumen de mediciones realizadas con el FWD (continuación).

\begin{tabular}{|c|c|c|c|}
\hline Punto & Fechas FWD & Estación & Región climática \\
\hline \multirow{5}{*}{$\begin{array}{c}\text { Punto } 20 . \\
\text { San Rafael - San Antonio }\end{array}$} & 08-08-02 & Lluviosa & \multirow{4}{*}{ Valle Central } \\
\hline & $14-10-04$ & Lluviosa & \\
\hline & $18-05-05$ & Lluviosa & \\
\hline & $28-07-06 *$ & Lluviosa & \\
\hline & $14-03-02$ & Seca & \multirow{5}{*}{ Valle Central } \\
\hline \multirow{5}{*}{$\begin{array}{l}\text { Punto } 21 . \\
\text { Alajuela - Itiquís }\end{array}$} & 01-10-03 & Lluviosa & \\
\hline & $11-10-04$ & Lluviosa & \\
\hline & 21-04-05 & Seca & \\
\hline & $10-07-06^{*}$ & Lluviosa & \\
\hline & $14-03-02$ & Seca & \multirow{5}{*}{ Valle Central } \\
\hline \multirow{4}{*}{$\begin{array}{c}\text { Punto } 22 . \\
\text { Itiquís - San Isidro }\end{array}$} & 01-10-03 & Lluviosa & \\
\hline & $11-10-04$ & Lluviosa & \\
\hline & $21-04-05$ & Seca & \\
\hline & $10-07-06^{*}$ & Lluviosa & \\
\hline \multirow{5}{*}{$\begin{array}{c}\text { Punto } 23 . \\
\text { Int. Ruta } 1 \text { - Naranjo }\end{array}$} & 07-08-02 & Lluviosa & \multirow{5}{*}{ Valle Central } \\
\hline & $21-10-03$ & Lluviosa & \\
\hline & $05-11-04$ & Lluviosa & \\
\hline & $27-04-05$ & Seca & \\
\hline & $11-07-06^{*}$ & Lluviosa & \\
\hline \multirow{3}{*}{$\begin{array}{c}\text { Punto } 24 . \\
\text { San Ramón- Puntarenas }\end{array}$} & $05-11-04$ & Lluviosa & \multirow{3}{*}{$\begin{array}{c}\text { Cordillera Guanacaste } \\
\text { Tilarán }\end{array}$} \\
\hline & 27-04-05 & Seca & \\
\hline & $18-07-06 *$ & Lluviosa & \\
\hline \multirow{3}{*}{$\begin{array}{l}\text { Punto } 25 . \\
\text { Florencio del Castillo }\end{array}$} & 26-11-04 & Lluviosa & \multirow{3}{*}{ Valle Central } \\
\hline & $22-04-05$ & Seca & \\
\hline & $14-07-06^{*}$ & Lluviosa & \\
\hline \multirow{3}{*}{$\begin{array}{c}\text { Punto } 26 . \\
\text { Próspero Fernández }\end{array}$} & $26-11-04$ & Lluviosa & \multirow{3}{*}{ Valle Central } \\
\hline & 29-03-05 & Seca & \\
\hline & $14-04-06 *$ & Lluviosa & \\
\hline \multirow{3}{*}{$\begin{array}{c}\text { Punto } 27 . \\
\text { Nicoya - Santa Cruz }\end{array}$} & $29-10-04$ & Lluviosa & \multirow{4}{*}{ Península Nicoya } \\
\hline & $26-04-05$ & Seca & \\
\hline & $19-07-06 *$ & Lluviosa & \\
\hline \multirow{3}{*}{$\begin{array}{c}\text { Punto 28. } \\
\text { Liberia - La Cruz }\end{array}$} & $28-10-04$ & Lluviosa & \\
\hline & $25-04-05$ & Seca & \multirow{2}{*}{$\begin{array}{l}\text { Golfo Nicoya Llanura } \\
\text { Guanacasteca }\end{array}$} \\
\hline & $18-07-06^{*}$ & Lluviosa & \\
\hline \multirow{3}{*}{$\begin{array}{l}\text { Punto } 29 . \\
\text { Arizona - La Irma }\end{array}$} & $28-10-04$ & Lluviosa & \multirow{3}{*}{$\begin{array}{l}\text { Golfo Nicoya Llanura } \\
\text { Guanacasteca }\end{array}$} \\
\hline & $25-04-05$ & Seca & \\
\hline & $18-07-06^{*}$ & Lluviosa & \\
\hline \multirow{3}{*}{$\begin{array}{c}\text { Punto } 30 . \\
\text { Cárcel (Puntarenas) - Caldera }\end{array}$} & 04-11-04 & Lluviosa & \multirow{3}{*}{$\begin{array}{c}\text { Golfo Nicoya Llanura Gua- } \\
\text { nacasteca }\end{array}$} \\
\hline & $26-04-05$ & Seca & \\
\hline & $20-07-06^{*}$ & Lluviosa & \\
\hline
\end{tabular}

* Nota: Las fechas marcadas con asteriscos no poseen información de la temperatura del pavimento en el momento de la realización de las mediciones, por lo cual fueron descartadas para el análisis. 
utiliza la Figura 4, conforme a lo establecido en el método de la (AASHTO, 1993).

Finalmente, la deflexión máxima corregida de la deflectometría de impacto es calculada utilizando la ecuación (2).

$$
\mathrm{d}_{\max 20^{\circ} \mathrm{C}}=\mathrm{d}_{\text {max ensayo }} \cdot \mathrm{F}_{\text {ajtemp }}
$$

donde:

$\mathrm{d}_{\max 20^{\circ} \mathrm{C}}$ deflexión corregida a la temperatura estándar de $20^{\circ} \mathrm{C}$

$\mathrm{d}_{\text {maxensayo }}$ deflexión medida a la temperatura superficial del pavimento en el ensayo

$\mathrm{F}_{\text {ajtemp }} \quad$ factor de ajuste por temperatura

\section{CONCLUSIONES}

Teniendo en consideración cada una de las regiones climáticas, se realizó un análisis estadístico de comparación de promedios de las deflexiones en las diferentes estaciones y fechas para cada uno de los puntos, obteniéndose las siguientes conclusiones.
1. La variación estacional de módulos para una estructura de pavimento se debe a cambios en el contenido de humedad de las capas granulares y suelos, por lo tanto se espera que aquellas estructuras que son sometidas a cambios más marcados de humedad presenten las variaciones más significativas. Por el contrario, zonas donde no existen cambios importantes en la humedad, no presentarán cambios importantes. Esta variación estacional de módulos se verá reflejada, con las deflexiones medidas por el equipo utilizado para valorar la capacidad estructural, llamado deflectómetro de impacto (FWD).

2. Con base en lo anterior y en el análisis realizado, se logró comprobar que los puntos ubicados en las zonas climáticas: Caribe, Caribe Sur y Caribe Norte, no presentan variaciones significativas en las deflexiones. Esto se puede atribuir a que en estas zonas se presentan once o doce meses de estación lluviosa, por lo tanto no existen variaciones importantes de humedad. Por su parte, la región Upala-Los Chiles, si bien presenta un mayor número de meses catalogados

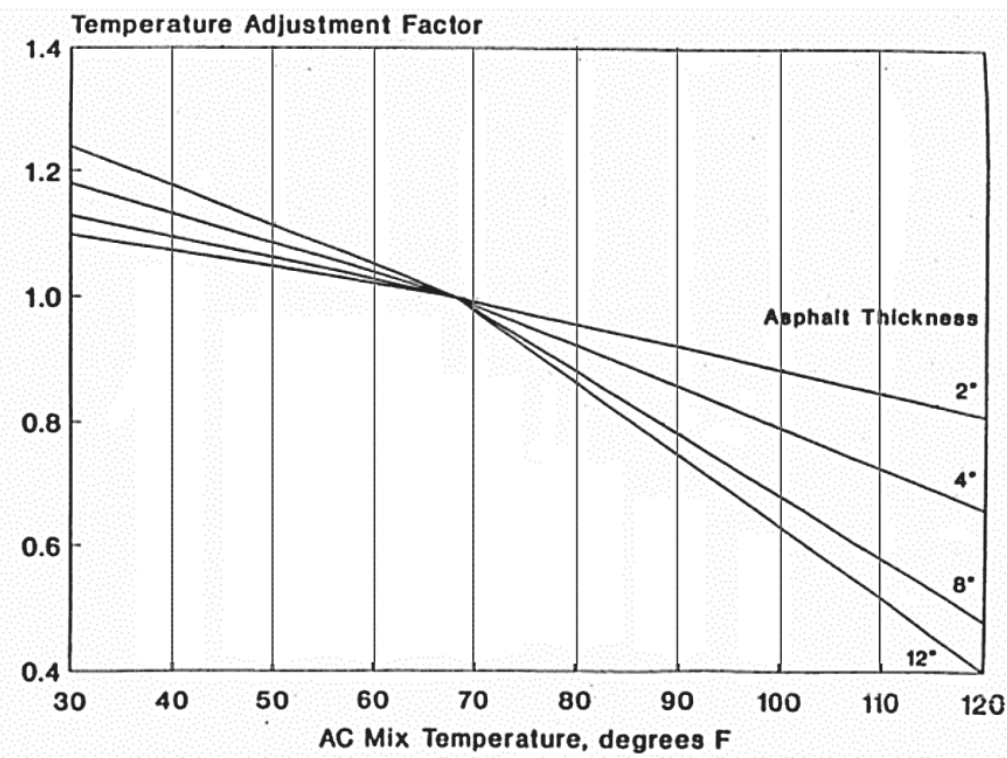

Figura 4. Factor de corrección por temperatura en función del espesor de la capa asfáltica. Fuente: (AASHTO, 1993). 


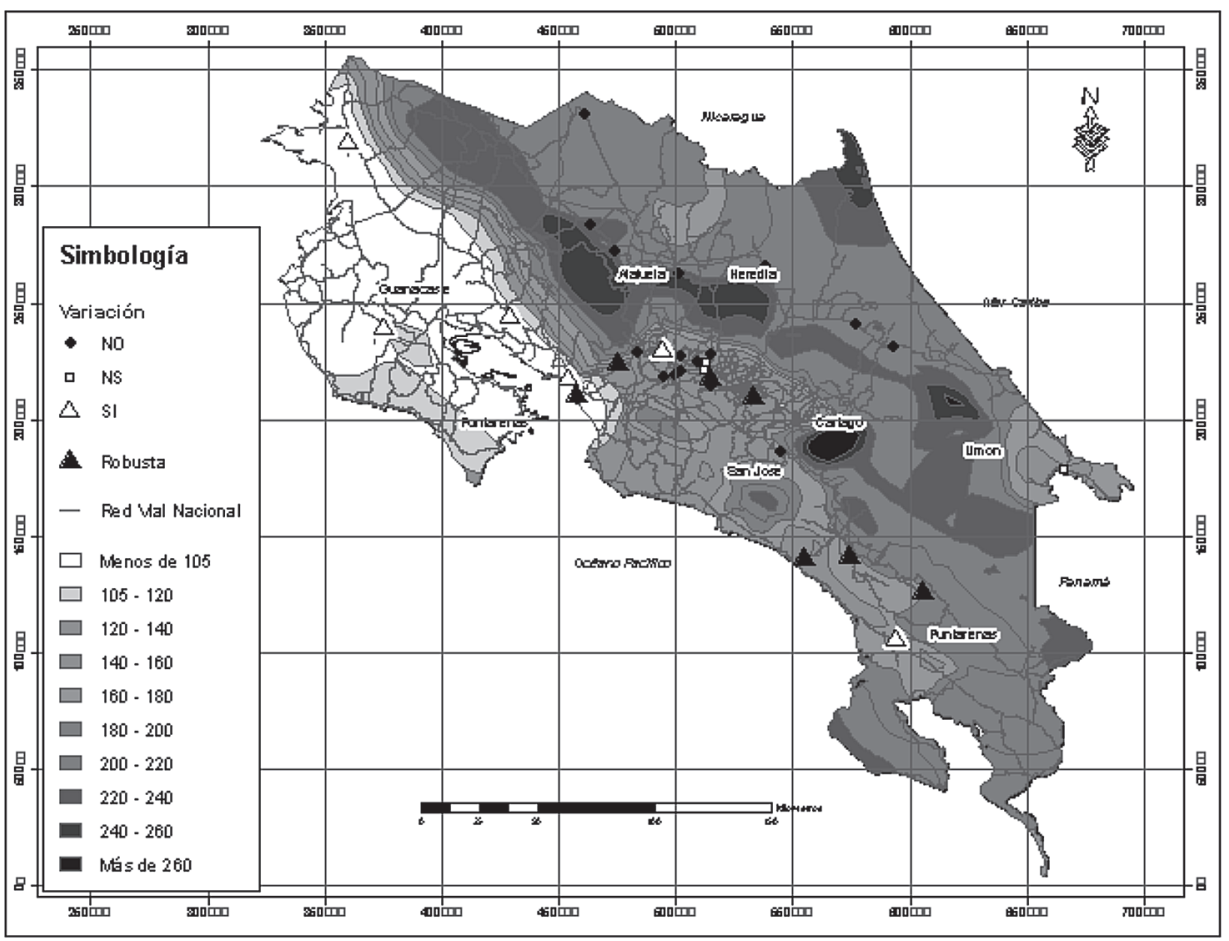

Figura 5. Ubicación de los puntos para los días de lluvia.

Fuente: (Orozco, 2006).

como secos (cuatro meses) la condición predominante en esta zona es de alta humedad, razón por la cual no se presentan variaciones significativas en las deflexiones.

3. El caso contrario ocurre en las regiones Golfo de Nicoya, Llanura Guanacasteca y Península de Nicoya, las cuales se caracterizan por la presencia de estaciones climáticas bien marcadas, donde se reflejan variaciones significativas de las deflexiones promedio. Por lo tanto y con base en lo anterior, se concluye que en estas zonas existe un efecto significativo de la estación climática seca o lluviosa, según corresponda.

4. En el caso específico de la Región Valle Central, dentro de los puntos analizados se presentan estructuras cuyas deflexiones son significativamente diferentes, así como estructuras para las cuales las deflexiones no lo son, siendo esta segunda condición la más generalizada; no obstante, para este segundo grupo sí se nota una tendencia en la reducción de las deflexiones en época seca respecto a la época lluviosa. Con base en lo anterior, se recomienda tomar en cuenta la estación climática para la medición de las deflexiones en el Valle Central.

5. Para la región Pacífico Central, el análisis muestra que no existen diferencias significativas entre las deflexiones medidas en época lluviosa y seca. Este resultado puede deberse a que solo se estudió una estructura con deflexiones muy bajas, las cuales tienden a no presentar diferencias entre la época lluviosa y época seca, por lo que para estructuras con mayores deflexiones podrían ser más evidentes las diferencias. Por lo anterior, se recomienda para esta región tomar en cuenta la estación 


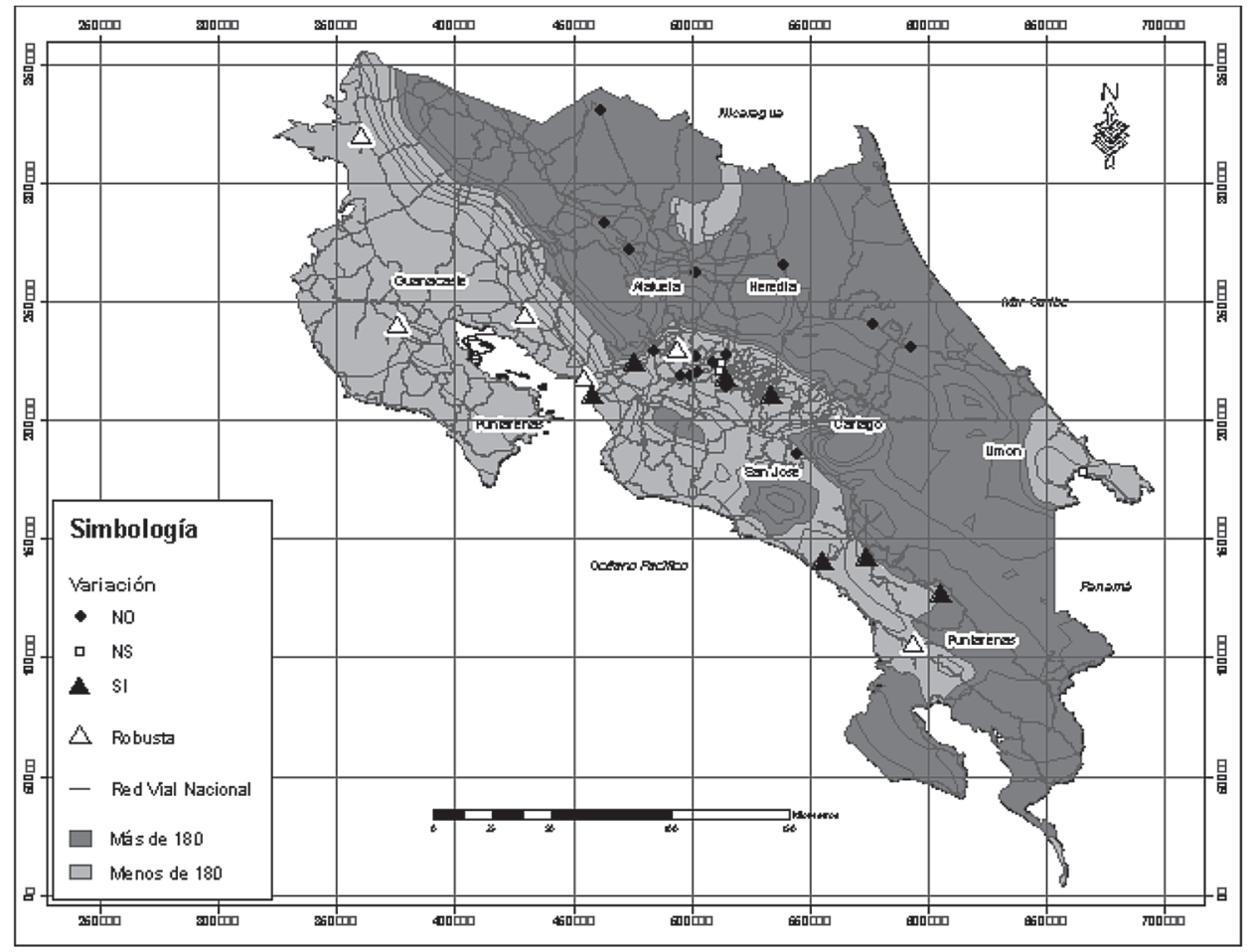

Figura 6. Zonas con más de 180 d de lluvia en el año.

Fuente: (Orozco, 2006).

durante las mediciones, hasta no verificar lo contrario con mayor número de puntos.

6. Para la región Los Santos se evaluó un punto ubicado en la Ruta 2, el cual no presenta diferencias significativas entre los promedios de deflexión para la época lluviosa y seca, sin embargo, por la variación en microclimas presentes en esta zona se recomienda tomar en cuenta la época del año en que se realizan las evaluaciones.

7. En el caso de la región Valle del General, dados los resultados se recomienda tomar en cuenta la época lluviosa para las evaluaciones de deflectometría.

8. De acuerdo con los resultados obtenidos, todas aquellas rutas con deflexiones menores a $25 \mathrm{~mm}^{-2}$, no presentan diferencias significativas entre la estación seca y lluviosa, indiferentemente de la Región en la que se encuentren. Por lo tanto, se puede concluir que para estructuras robustas la variación estacional de módulos no es significativa desde el punto de vista de las deflexiones medidas mediante el FWD. En la Figura 5 estas se distinguen con triángulos negros y están ubicadas, en su mayoría, en el Valle Central y en la Zona Sur.

9. Comparando los resultados obtenidos con la precipitación (días de lluvia en el año) para los puntos evaluados, se concluye que aquellos puntos que no presentaron diferencias significativas entre los resultados, son los que se encuentran en zonas con más de $180 \mathrm{~d}$ de lluvia del año (50 \% días de lluvia). Esta información se puede observar en la Figura 6.

\section{RECOMENDACIONES}

1. En las zonas climáticas: Caribe Alta, Norte Alta, Pacífico Sur, Talamanca y Volcánica Central Pacífica no existen puntos de 
evaluados, por lo tanto se recomienda incluir algunos para analizar el comportamiento de las deflexiones en estas zonas a lo largo del año.

2. Es necesario que los puntos que vayan a ser sujetos de estudio, cuenten con información lo más completa posible sobre sus características básicas (temperatura, precipitación, estructura -espesor y materiales-, tránsito).

3. Es importante que se dé un seguimiento sistemático a cada punto de evaluación, de manera que se monitoreen periódicamente las deflexiones, así como cualquier cambio que pueda influir en la respuesta estructural del pavimento.

4. Para un futuro estudio sería recomendable realizar un análisis que permita correlacionar las estructuras -espesor y materiales- con la variación de las deflexiones, bajo las diferentes condiciones de temperatura y humedad que se presenten.

5. Es conveniente incluir en estudios posteriores, retrocálculos de módulos que permitan concluir (según la estación climática del año) cómo varían los módulos de los distintos materiales dentro de la estructura del pavimento.

\section{REFERENCIAS BIBLIOGRÁFICAS}

American Association of State Highway and Transportation Officials (AASHTO). (1993). AASHTO Guide for design of pavement structures 1993. Washington, D. C.

Asphalt Institute. (s.f.) Asphalt overlays for highway and street rehabilitation. Manual Series No. 17 (MS-17). Lexington, Kentucky.

Badilla, G. \& Sibaja, D. (2008). Análisis de resultados de deflectometría de impacto
(FWD) para la evaluación de la respuesta estructural de los pavimentos para diferentes épocas del año en la red vial de Costa Rica. San José: Universidad de Costa Rica, Unidad de Investigación en Infraestructura Vial. Laboratorio Nacional de Materiales y Modelos Estructurales.

Orozco, E. (2006). Zonificación climática de Costa Rica la gestión de obras viales. Proyecto para optar por el grado de licenciatura en Ingeniería Civil, Facultad de Ingeniería, Universidad de Costa Rica, San José, Costa Rica.

\section{SOBRE EL AUTOR Y LA AUTORA}

\section{Gustavo A. Badilla Vargas}

Ingeniero civil. Licenciado en Ingeniería Civil de la Universidad de Costa Rica

Laboratorio Nacional de Materiales y Modelos Estructurales

Unidad de Investigación en Infraestructura Vial (UI)

Universidad de Costa Rica, Ciudad Universitaria Rodrigo Facio,

San Pedro de Montes de Oca, Costa Rica

Teléfono: (506) 2511-4994

Facsímil: (506) 2511-4442

Correo electrónico: gustavo.badilla@ucr.ac.cr

\section{Denia Sibaja Obando}

Ingeniera civil.

Licenciada en Ingeniería Civil de la Universidad de Costa Rica

Soporte técnico, caminos

Proyecto Hifroeléctrico Ampliación Cachí

Cachí, Cartago

Teléfono: (506) 8847-3432 / 2558-1500 ext.9003

Correo electrónico: DeSiba@ice.go.cr 
